\title{
Kateřina Nekolová*
}

\section{Nachhaltigkeit als Programm}

\author{
Ein Interview mit der tschechischen AG „Nachhaltigkeit in Bibliotheken“(Udržitelnost v \\ knihovnách)
}

https://doi.org/10.1515/bfp-2021-0071

Zusammenfassung: Im Jahr 2019 bildete sich in Tschechien eine Gruppe aus Bibliothekarinnen und Bibliothekaren, die sich dem Thema Nachhaltigkeit zu widmen begannen. Im Jahr 2021 ist Nachhaltigkeit das offizielle Thema des tschechischen Bibliotheksverbands. Es findet eine Reihe von Veranstaltungen statt, von denen die wichtigste der ganzjährige Bildungszyklus ist.

Schlüsselwörter: Nachhaltigkeit; Bibliotheken; Tschechien

\section{Sustainability as a Programme \\ An Interview with the Czech Collective Sustainability in Libraries (Udržitelnost v knihovnách)}

Abstract: In 2019, a group of librarians was established in the Czech Republic, who began to dedicate themselves to the topic of sustainability. In 2021, sustainability will be the official theme of the Czech Library Association. A number of events take place, the most important of which is the year-round educational cycle.

Keywords: Sustainability; libraries; Czech Republic

Im Jahr 2019 begann eine unabhängige Arbeitsgruppe (AG) tschechischer Bibliothekarinnen und Bibliothekare, sich für das Thema Nachhaltigkeit zu engagieren. Aus zunächst einigen einzelnen Veranstaltungen entwickelten sich landesweite Aktivitäten. Die Gruppe ist bemüht, Nachhaltigkeit auch in Zukunft zu einem selbstverständlichen Thema für die Bibliotheken zu machen, da diese mit ihrem Grundprinzip des Teilens an sich schon nachhaltige Ziele verfolgen. Im folgenden Interview beantworten Mitglieder der AG „Nachhaltigkeit in Bibliotheken“ (NiB) ${ }^{1}$ Fragen zu ihren

1 https://udrzitelna.knihovna.cz/. Zur Zeit gehören zu den aktivsten Mitgliedern: Eliška Bartošová (Brünn), Veronika Chruščová und Ondřej Hudeček (Prag), Martina Košanová (Pilsen), Martin Ďurkáč (Zlín). Die Zusammenarbeit erstreckt sich daher über die gesamte Tschechische Republik.

*Kontaktperson: Kateřina Nekolová, katerina.nekolova@nkp.cz
Aktivitäten. Die Fragen stellte Kateřina Nekolová (KN) aus dem Bibliotheksinstitut in Prag.

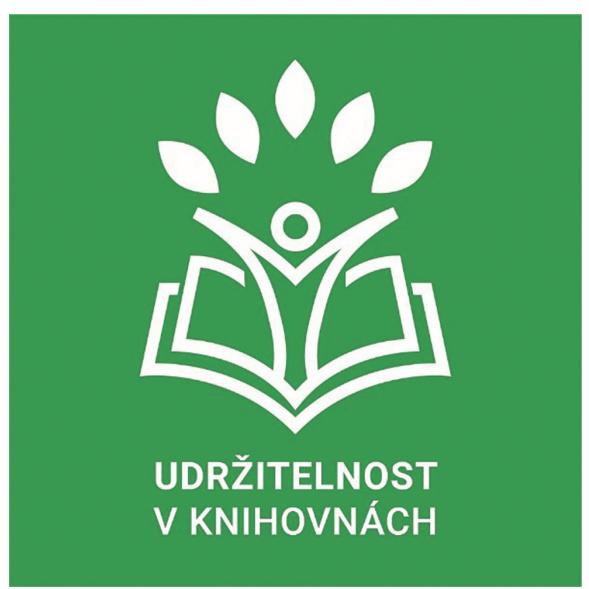

Abb. 1: Logo der AG „Udržitelnost v knihovnách“(Nachhaltigkeit in Bibliotheken $)^{2}$

KN: Nehmen Sie Unterschiede bei der Beschäftigung mit dem Thema Nachhaltigkeit in Tschechien und den deutschsprachigen Ländern wahr?

NiB: Aus internationalen fachlichen Kontakten haben wir den Eindruck gewonnen, dass in den deutschsprachigen Ländern diese Haltung eine viel längere Tradition und eine stabilere Basis hat. Vielleicht gibt es dort auch ein größeres Entgegenkommen seitens der Öffentlichkeit, das Thema wird besser angenommen. Im tschechischen Bibliothekswesen stehen wir erst am Anfang, die ersten Materialien werden erarbeitet und Initiativen Einzelner und konkreter Bibliotheken werden geteilt. Diese Phase ist sehr interessant und die Coronapandemie gibt uns die Möglichkeit, mit mehr Rücksicht auf Nachhaltigkeit zum normalen Betrieb zurückzukehren.

$K N$ : Was waren die Impulse dafür, dass sich das tschechische Bibliothekswesen zentral dem Thema Nachhaltigkeit zu widmen begann?

2 https://udrzitelna.knihovna.cz/, udrzitelna@knihovna.cz. 
NiB: Impulse gab es gleich mehrere. Einige Bibliothekarinnen und Bibliothekare beschäftigen sich schon längere Zeit mit Themen, die mit dem Klima, der Umwelt oder sozialer Verantwortung zusammenhängen; jetzt fanden sie in der Nachhaltigkeit ein gemeinsames, übergreifendes Konzept. Einige begannen sich 2019 privat der Bewegung „Fridays for Future“ ${ }^{3}$ oder den Aktivitäten der Universitäten für das Klima ${ }^{4}$ anzuschließen. Dabei erkannten sie die wichtige Rolle, die die Bibliotheken beim Übergang $\mathrm{zu}$ einer nachhaltigeren Einstellung, zum Beispiel durch Bildungsangebote oder ihre Rolle als Vorbild, übernehmen können.

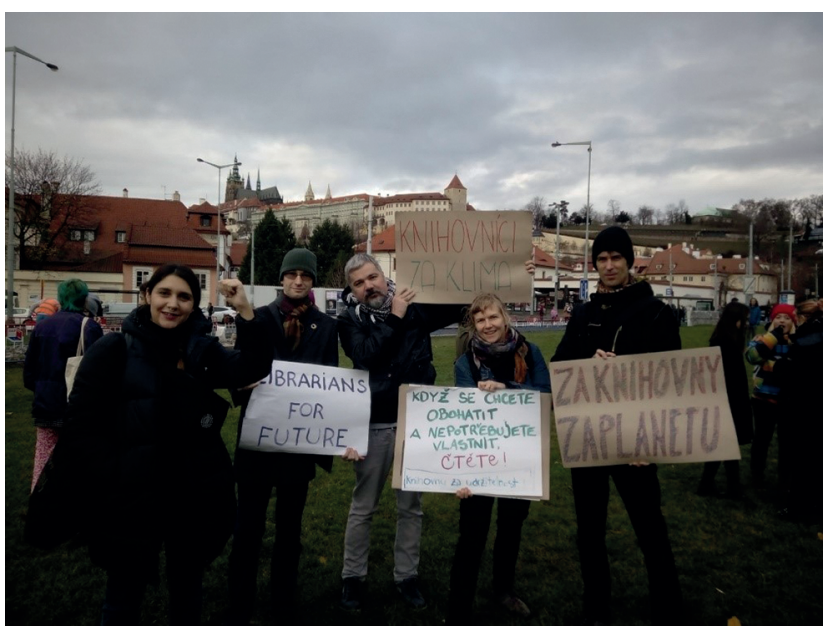

Abb. 2: Die AG „Nachhaltigkeit in Bibliotheken“ beteiligt sich an der weltweiten Klimademonstration 2019 als „Librarians for Future“, (c) Privat

Es gibt einige längerfristige Bibliotheksprojekte, deren Aktivitäten sich auch um das Thema Nachhaltigkeit drehen. Zum Beispiel fanden im Rahmen des Projekts „Soziale Innovation in Bibliotheken “5 Vorträge für die Bibliothekarinnen und Bibliothekare des Bezirks Ústí nad Labem über die Möglichkeiten der Transformation der Kohleregion statt. Einige von ihnen schlossen sich internationalen Aufklärungsaktivitäten in diesem Bereich an. Auch auf dem akademischen Boden stieg das Bewusstsein für dieses Thema. Es gibt einzelne Interessierte, die sich dem Thema im Rahmen ihres Studiums des Bibliothekswesens widmen, auch auf der Ebene einer Promotion. Parallel dazu gibt es in Tschechien auch Bibliotheken, die sich schon viele Jahre mit Ökologie und Umweltfragen beschäftigen, oder Bibliotheken, die einen Umbau ihrer Gebäude mit

3 https://fridaysforfuture.org/.

4 https://univerzityzaklima.cz/.

5 https://komunitni.knihovna.cz/.
Hinblick auf eine bessere Energiebilanz planen. Gleichzeitig haben wir eine Reihe Bibliotheken, deren Programme und Veranstaltungen als sehr progressiv zu bezeichnen sind und im Einklang mit den Prinzipien einer nachhaltigen Gesellschaft stehen. Im Jahr 2019 trafen sich zum ersten Mal Bibliothekarinnen und Bibliothekare mit dem Direktor des Informationszentrums der Vereinten Nationen in Prag, Michal Broža, um darüber zu diskutieren, wie man mit den UN-Nachhaltigkeitszielen (Sustainable Development Goals, SDGs) in den Bibliotheken in Tschechien arbeiten kann. Es folgte ein Treffen im Prager Büro der UN und die Gründung einer unabhängigen informellen Arbeitsgruppe, die sich seither diesem Thema widmet.

KN: Gab es schon früher in den Bibliotheken ähnliche Aktivitäten, die unmittelbar mit den Nachhaltigkeitszielen oder mit dem Umweltschutz zusammenhingen?

NiB: Natürlich veranstalteten viele Bibliotheken regelmäßig Aktivitäten, die auf lokaler Ebene der Gemeinschaft oder Umwelt nützlich waren. Sie brachten sie vielleicht nur nicht mit den aktuellen Prinzipien der Agenda 2030 der Vereinten Nationen oder der entsprechenden Terminologie in Verbindung. Die Tschechische Republik hat das dichteste Bibliotheksnetz der Welt. ${ }^{6}$ Die Bibliotheken sind ein neutraler öffentlicher Ort, eine vertrauenswürdige Institution, die als Partner für die Ziele im Bereich der Nachhaltigkeit fungieren kann. Bibliotheken sind ein Pfeiler der Bürgergesellschaft, der Bildung und Kultur, wie aus dem aktuellen Konzept zur Entwicklung der Bibliotheken in der Tschechischen Republik für den Zeitraum 2021-2027 mit Ausblick bis $2030^{7}$ hervorgeht.

Wir haben sehr aktive Bibliotheken. Eine von ihnen ist die Öffentliche Bibliothek der Stadt Strakonice - die Schmidinger-Bibliothek, ${ }^{8}$ die schon seit 2005 eine Beratungsstelle für Non-Profit-Organisationen und ökologische Belange betreibt. Der Bibliothekar Jan Juráš kann nicht nur theoretische Fragen beantworten, sondern auch bei der Lösung konkreter Situationen beraten. Es gibt dort auch einen thematischen Bestand entsprechender Literatur.

Im Jahr 2012 entstand das Studentenprojekt Grüne Bibliothek ${ }^{9}$ im Rahmen eines Seminars an der Masaryk Universität in Brünn. Auf der gleichnamigen Webseite

6 Diese Tatsache wurde auch in ausländischen Medien erwähnt: https://www.nytimes.com/2016/07/22/world/what-in-the-world/why -libraries-are-everywhere-in-the-czech-republic.html.

7 Národní knihovna České republiky (2020).

8 https://knih-st.cz/.

9 http://zelena.knihovna.cz/. 
kann man nicht nur allgemeine Empfehlungen finden, sondern auch testen lassen, wie „grün“, d.h. nachhaltig der Betrieb der eigenen Bibliothek ist.

KN: Sie haben eine Umfrage unter den tschechischen Bibliotheken durchgeführt. Was waren die Ergebnisse?

NiB: Im Herbst 2020 fand eine Online-Umfrage unter den Bibliotheken statt. An der Vorbereitung und Auswertung der Fragebögen hat hauptsächlich unsere Kollegin Eliška Bartošová gearbeitet. Der Fragebögen wurde per E-Mail über die fachlichen Netzwerk (ca. 1000 Bibliotheken) und über die Kreisbibliotheken verbreitet. Insgesamt haben wir 345 ausgefüllte Formulare auswerten können.

Der Fragebogen lag in zwei Versionen vor - für kleine und für große Bibliotheken, wobei es keine strikte quantitative Einteilung gab. In der Tschechischen Republik funktioniert ein Bibliotheksnetz, in dem die Bezirksbibliotheken bestimmten lokalen Bibliotheken Unterstützung gewähren, und diese kümmern sich dann methodisch um die kleinen Bibliotheken. Dank dieses Systems konnten wir größere Bibliotheken direkt kontaktieren und sie bitten, den Fragebogen auszufüllen sowie ihn an kleinere Bibliotheken weiterzuleiten. Der Fragebogen wurde daher von 209 Bibliotheken ausgefüllt, die sich als klein bezeichnet haben, und von 136 Bibliotheken, die sich als groß deklariert haben.

Der Fragebogen bestand aus drei Teilen. Im ersten Teil wollten wir wissen, wie sich die Bibliotheken schon heute an einer nachhaltigen Entwicklung beteiligen. Dazu gab es eine Reihe von Aussagen, denen die Befragten zustimmen oder die sie ablehnen konnten. Der zweite Teil zielte auf den Willen, vermehrt Aktivitäten in dieser Richtung zu gestalten. Hier wurden Vorschläge aufgelistet, wie die Bibliotheken ihre Tätigkeit im Bereich der Nachhaltigkeit erweitern könnten und die Befragten sollten für jeden einzelnen Vorschlag angeben, ob er in der jeweiligen Bibliothek umsetzbar oder überflüssig ist, ob sie ihn schon ausprobiert haben oder vorhaben, ihn umzusetzen. Im letzten Teil der Umfrage wollten wir von den Bibliotheken wissen, was sie zur Umsetzung der Vorschläge bräuchten und was sie interessieren würde.

Im ersten Teil haben wir vorausgesetzt, dass sich die Bibliotheken als Institutionen bereits an der Erreichung der UN-Nachhaltigkeitszielebeteiligen. Die Tatsache, dass sie einen gleichberechtigten Zugang zu Informationen sicherstellen, galt als Grundvoraussetzung. Darüber hinaus wurde nach der Umsetzung weiterer Nachhaltigkeitsziele der Agenda 2030 gefragt.

Am häufigsten stimmten die Bibliotheken folgenden Aussagen zu:
- Bibliotheken bieten eine ruhige Atmosphäre, jeder kann sie aufsuchen, auch um sich einfach nur dort aufzuhalten, in Ruhe und sicherer Umgebung abzuschalten und sich zu erholen (entspricht Ziel 3 der SDGs - Gesundheit und Wohlergehen).

- Bibliotheken sind kein kommerzieller Raum, es geht hier nicht um Besitz. Dank dessen haben die Menschen freien Zugang zu Informationen, Bildung und Unterhaltung (entspricht Ziel 1 - Ende der Armut und Ziel 10 - Weniger Ungleichheit).

- Bibliotheken sind Orte des lebenslangen Lernens und der Bildung, die allen zugänglich sind (entspricht Ziel 4 - Hochwertige Bildung).

- Bibliotheken bieten die Möglichkeit einer sinnvollen Freizeitgestaltung ohne Konsumzwang (entspricht Ziel 12 - Nachhaltige Konsum- und Produktionsmuster).

Die größten Defizite sahen die Bibliotheken dagegen in der beschränkten Möglichkeit, als Vorbild für den sparsamen Umgang mit Umweltressourcen zu dienen. Dies ist verständlich, sie haben nur wenige Möglichkeiten, z. B. die Energiebilanz der Gebäude, in denen sie untergebracht sind, zu beeinflussen. Trotzdem haben die Bibliotheken aber die Möglichkeit, das Bewusstsein ihres Publikums mit ihrer Aufklärungs- und Informationsarbeit weiter zu entwickeln. Auch wenn keine Perfektion erreicht werden kann, wird ein Denkprozess in Gang gesetzt und man sucht nach Wegen, wie man seinen Beitrag leisten kann. Und das kann bei den Nutzern zu einem ähnlichen Prozess des Nachdenkens führen. Außerdem beruhen die Bibliotheken an sich auf dem Prinzip des Teilens und helfen dadurch, Ressourcen zu sparen. Die an der Umfrage beteiligten Bibliotheken gaben eine Vielzahl von Aktivitäten an, die sie selbst schon unternommen hatten, z.B. Saatgut-Tauschbörsen, eine Nachbarschaftsuniversität oder Filmabende mit ökologischer Thematik.

Was die Aktivitäten betrifft, die die Bibliotheken gerne ausbauen würden, handelt es sich vor allem um die Arbeit mit dem Bibliotheksbestand, außerdem darum, durch eine überzeugende Umsetzung nachhaltiger Ideen als Vorbild $\mathrm{zu}$ dienen, ihre Bildungsangebote zu erweitern, Hilfe bei „ökologischer Trauer“ (,ecological grief“"10) oder auch erweiterte Informationsdienste anzubieten.

Bei beiden Bibliothekstypen galten als bevorzugte Themen im Hinblick auf die Nachhaltigkeitsziele „Gesundheit und Wohlbefinden“, gefolgt von „Hochwertiger Bil-

10 https://en.wikipedia.org/wiki/Ecological_grief. 
dung“ und „Nachhaltige Gemeinden und Städte“ - also Themen, die jeden von uns betreffen.

Zum Abschluss der Befragung sollten die Bibliotheken angeben, was ihnen am meisten helfen würde bei der Umsetzung der Nachhaltigkeitsziele. Das waren: Teilen von Erfahrungen und Inspiration, Verbindung zu Experten, Anleitungen, wie die Bibliothek als Vorbild dienen kann, sowie das Teilen ausgearbeiteter Materialien und Inhalte.

Die Ergebnisse dieser Umfrage sind eine wertvolle Orientierung bei der Planung von Bildungsveranstaltungen. Auf der anderen Seite wollen wir die Themen, die von den Teilnehmern nicht als interessant eingestuft wurden, nicht gänzlich außer Acht lassen. Ein möglicher Grund für die Nichtnennung könnte nämlich gerade ein zu niedriges Bewusstsein für die gegebene Problematik oder deren Komplexität sein.

KN: Welche Rolle spielt die Einbindung des nationalen Verbands der Bibliothekare und Informationsdienstleister SKIP ${ }^{11}$ bei der Durchsetzung des Themas Nachhaltigkeit in den tschechischen Bibliotheken?

NiB: Es ist sicher von Vorteil, dass der SKIP Nachhaltigkeit als zentrales Thema des Jahres 2021 ausgerufen hat. Jedes Jahr wird ein zentrales Thema festgelegt, dem dann im tschechischen Bibliothekswesen während des ganzen Jahres vermehrt Aufmerksamkeit gewidmet wird. Im letzten Jahr war es das Thema Bildung, davor lokale Geschichte. SKIP ist gleichzeitig der offizielle Veranstalter des ganzjährigen Weiterbildungsprogramms zum Thema Nachhaltigkeit. Dadurch hat das Programm nicht nur eine geförderte Unterstützung, sondern vor allem eine gesicherte Qualität. Das Programm wurde auch durch die offizielle Schirmherrschaft des Ministeriums für Kultur, des Umweltministeriums, des Informationszentrums der UN in Prag und der Tschechischen Kommission der UNESCO gefördert. Wir schätzen es sehr, dass diese bedeutenden Institutionen das Potenzial der Bibliotheken bei der Durchsetzung einer nachhaltigen Haltung und Lebensweise anerkennen.

$\boldsymbol{K N}$ : Wie sieht es im Umfeld von Berufsausbildung und Studium aus? Besteht dort Interesse an dem Thema?
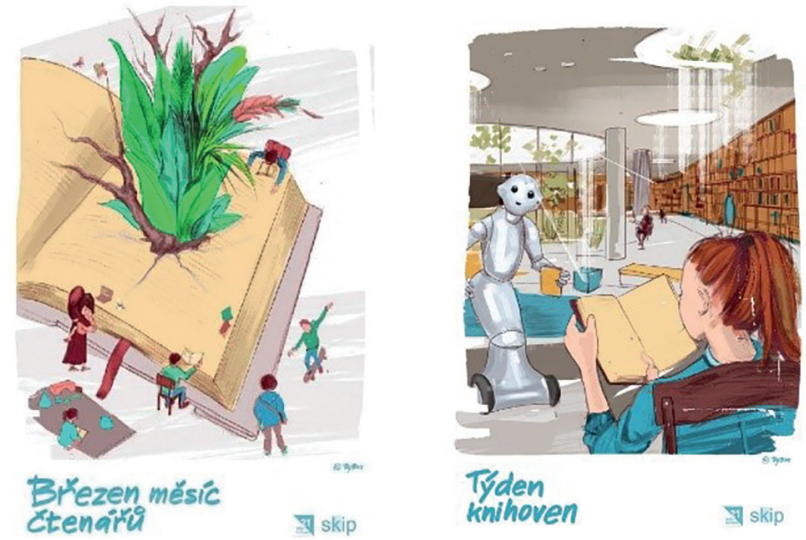

Abb. 3 und 4: Plakate des Verbands der Bibliothekare und Informationsdienstleister SKIP zu regelmäßigen Bibliotheksveranstaltungen zum Thema Nachhaltigkeit: März - Monat der Leser (Březen měsíc čtenářů) und Bibliothekswoche (Týden knihoven), @Toy_Box

NiB: Auf Grundlage der Zusammenarbeit des Prager Verbandes SKIP und des Instituts für Informationsstudien und Bibliothekswesen der Philosophischen Fakultät der Karlsuniversität entstanden zu Beginn des Jahres 2021 zwei Webinare und zwei Podcasts. Ursprünglich war eine ganztägige Konferenz zum Thema geplant, aufgrund der epidemiologischen Situation musste das Vorhaben jedoch den Bedingungen angepasst werden, und so fanden statt einer großen Veranstaltung in der Nationalbibliothek mehrere kleine Online-Veranstaltungen statt. $\mathrm{Zu}$ den Themen gehörten:

- Veranstaltungen mit Umweltthematik für Kinder in Bibliotheken, ${ }^{12}$

- Ökologische Aktivitäten in der Praxis der Bibliotheken, ${ }^{13}$

- Literatur für Kinder und Erwachsene zum Thema Ökologie ${ }^{14}$ und nachhaltiger Betrieb der Bibliotheken.

Die Weiterbildungsveranstaltungen wurden sehr gut angenommen und werden für die weitere Nutzung zugänglich gemacht. Es wurde in Betracht gezogen, dass die Ergebnisse gleich zu Beginn des Jahres vorliegen und so den Kolleginnen und Kollegen in der ganzen Republik langfristig als Anhaltspunkte dienen können.

In letzter Zeit nimmt auch das Interesse an Abschlussarbeiten zu, die mit dem Thema Nachhaltigkeit zusammenhängen.

12 https://www.youtube.com/watch?v=25hngpRr92w.

$13 \mathrm{https} / / /$ www.youtube.com/watch?v=lYHjsVbtgsg.

14 https://soundcloud.com/skippraha/environmetalni-literatura-pr o-knihovny. 
$K N$ : Mit wem arbeiten Sie noch zusammen?

NiB: In bedeutendem Maße hat sich die internationale Zusammenarbeit entwickelt. SKIP ist Mitglied der neu entstandenen IFLA-Sektion Environment, Sustainability and Libraries ${ }^{15}$ (ENSULIB). Durch die Zusammenarbeit mit SKIP stehen wir in Kontakt mit der Sekretärin dieser Sektion, Petra Hauke, die auch in einer kurzen Videopräsentation die Teilnehmerinnen und Teilnehmer unseres Auftaktseminars begrüßt hat. Unser Kollege Michal Hokynek übersetze die Green Library Checklist ${ }^{16}$ ins Tschechische.

Außerdem ist SKIP in die Expertengruppe European Library Sustainable Development Implementation and Assessment (ELSIA) ${ }^{17}$ unter dem europäischen Verband EBLI$\mathrm{DA}^{18}$ eingebunden, wo Eliška Bartošová aus Brünn als Delegierte auch unsere Arbeitsgruppe vertritt.

Von großer Bedeutung ist für SKIP die Zusammenarbeit mit dem Informationsbüro der Vereinten Nationen für die Tschechische Republik. Ihr Leiter Michal Broža arbeitet seit längerer Zeit eng mit SKIP zusammen, beteiligt sich an der Vorbereitung von Veranstaltungen, nimmt an Diskussionen teil und tritt bei Vorträgen für Bibliothekarinnen und Bibliothekare auf. Dank ihm bekommen wir nicht nur eine sehr fachliche Sicht auf die Nachhaltigkeitsziele, sondern auch wichtige Kontakte und wertvolles Feedback.

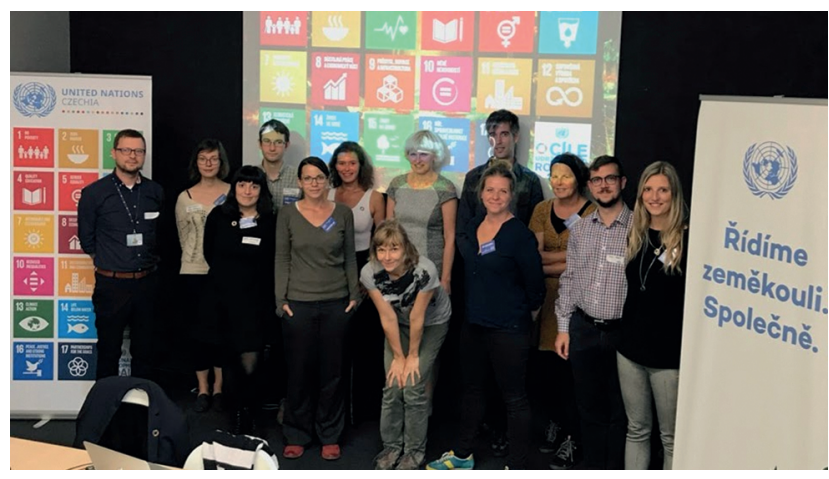

Abb. 5: Große Unterstützung erfährt die AG im Prager Büro der UN, (C) Privat

Außer dem SKIP ist auch der Verband der Bibliotheken der Tschechischen Republik (Sdružení knihoven ČR SDRUK) ${ }^{19}$ einer unserer Partner. Darüber hinaus gibt es
Kontakte und Zusammenarbeit mit den Initiativen Univerzity za klima ${ }^{20}$ (Universitäten für das Klima) und Učitelé za klima $^{21}$ (Lehrer für das Klima).

Auch mit Non-Profit-Organisationen arbeitet SKIP zusammen. Wir hoffen, dass es der Bibliothek Jiří Mahen ${ }^{22}$ in Brünn gelingt, Fördermittel für ein Projekt in Zusammenarbeit mit der Organisation Lipka, ${ }^{23}$ einer Organisation in der Tschechischen Republik, die sich mit der Erziehung, Bildung und Aufklärung im Bereich der Umwelt beschäftigt, zu bekommen. Das Ziel des Projekts besteht in der Umsetzung von Informations- und Bildungsveranstaltungen zu Themen wie der Abschwächung der Auswirkungen des Klimawandels und der Anpassung an den Klimawandel auf lokaler Ebene. Gegenstand des Projekts ist das Erarbeiten medialer Materialien, fachlicher und populärwissenschaftlicher Publikationen, das Organisieren von Schulungen, Seminaren, Workshops und anderen Aufklärungsaktivitäten und -kampagnen, die die genannten Themen auf lokaler Ebene ansprechen.

Darüber hinaus veröffentlicht die Arbeitsgruppe in der Fachzeitschrift Čtenář (Der Leser) im Laufe des Jahres immer wieder Artikel zum Thema Nachhaltigkeit.

\section{KN: Welche Aktivitäten haben schon stattgefunden?}

NiB: Am 25. Januar 2021 wurde mit dem Webinar „Nachhaltigkeit in Bibliotheken" das Jahr der Nachhaltigkeit in Bibliotheken offiziell eröffnet. Die Arbeitsgruppe hatte SKIP ein ganzjähriges Weiterbildungsprogramms als „Bildungszyklus“ vorgeschlagen. SKIP ist der offizielle Veranstalter und unterstützt das Programm finanziell, während die AG das Programm vorbereitet. Das Webinar verfolgten live fast 300 Teilnehmer aus verschiedenen Bibliotheken unterschiedlicher Größe, aktuell wurde es schon über $1000 \mathrm{Mal}$ angeschaut. Es gab darin auch einen Beitrag von Michal Broža, dem Direktor des Informationszentrums der UN in Prag, und Hana Matulová aus der Bibliothek in Kořenec, die aufgezeigt hat, wie auch eine kleine Bibliothek in einem kleinen Ort mit nicht einmal 400 Einwohnern das Thema Nachhaltigkeit in die Gemeinschaft einbringen kann. Am Ende wurden alle Kommunikationskanäle vorgestellt, über die man das Team der AG „Nachhaltigkeit in Bibliotheken“ verfolgen sowie Informationen einholen oder sich einbringen kann.

20 https://univerzityzaklima.cz/.

21 https://www.facebook.com/U\%C4 \%8Ditel\%C3 \%A9-za-klima-2 269069926515424/.

22 https://www.gotobrno.cz/de/ort/jiri-mahen-bibliothek-knihovna -jiriho-mahena/.

23 https://www.lipka.cz/lipka-de.

政
15 https://www.ifla.org/environment-sustainability-and-libraries.

$16 \mathrm{https} / / /$ www.ifla.org/publications/node/12621? og=479.

17 http://www.eblida.org/about-eblida/expert-groups/eu-librariessustainable-development-implementation-assessment.html.

18 http://www.eblida.org/.

19 https://sdruk.cz/. 
Auf der Facebookseite „Udržitelnost v knihovnách“ (Nachhaltigkeit in Bibliotheken) ${ }^{24}$ findet man nützliche Informationen, Tipps, Einladungen usw., es gibt Raum um sich mitzuteilen und mit Kolleginnen und Kollegen oder Experten darüber zu diskutieren, was die Bibliotheken aktuell interessiert. Die Webseite „Udržitelnost v knihovnách“ (Nachhaltige Bibliothek) ${ }^{25}$ hat eine Seite „Für Partner“, wo erklärt wird, warum und wie das Thema Nachhaltigkeit mit den Bibliotheken zusammenhängt. Bibliotheksbeschäftigte können potenzielle Partner auf die Seite verweisen, bevor sie mit ihnen ein Treffen oder eine Zusammenarbeit vereinbaren. Die Sektion „Für Bibliotheken“ ist ein Wegweiser für Informationen, Quellen und Inspiration. Neben einer Karte der Bibliotheken, die sich für das Thema Nachhaltigkeit interessieren, und einem Link zu einer Webseite mit erfolgreichen Beispielen finden die Bibliotheken hier auch einen Link zu dem öffentlichen Webdienst Trello. ${ }^{26}$ Auf dieser Pinnwand kommen allmählich immer mehr Beispiele für gelungene Umsetzung nachhaltiger Projekte zusammen, Informationsquellen, Übersichten über fertige Materialien und Unterlagen, aber auch über Fördermöglichkeiten für Bibliotheken.

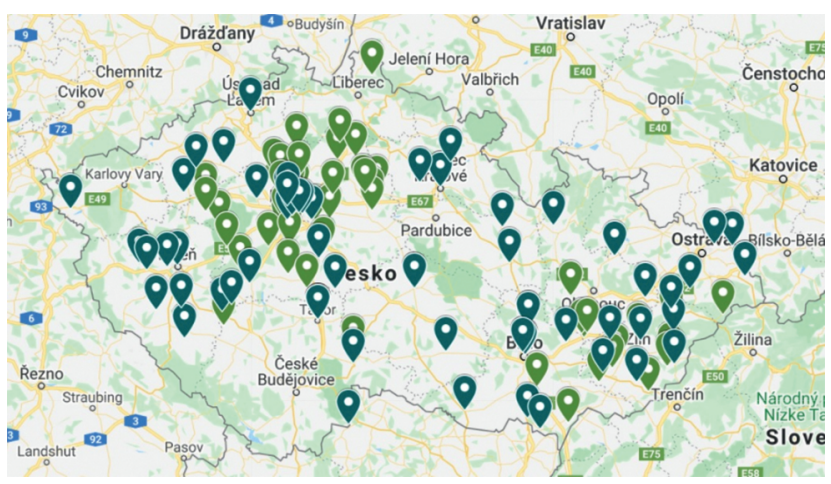

Abb. 6: Karte der Bibliotheken, die sich für das Thema Nachhaltigkeit interessieren. Hellgrün: Kleine Bibliotheken, Dunkelgrün: Größere und große Bibliotheken ${ }^{27}$

KN: Ihre Arbeitsgruppe ein Weiterbildungsprogramm zum Thema Nachhaltigkeit erarbeitet, das über das ganze Jahr laufen soll. Wie ist das geplant?

NiB: Das Programm sieht für 2021 sieben Webinare und drei praktische Workshops sowie eine Abschlusskonferenz vor. Die Anmeldungen der Teilnehmer erfolgen im Voraus

24 https://www.facebook.com/udrzitelnostvknihovnach/. 25 https://udrzitelna.knihovna.cz/.

26 https://trello.com/b/eHhIVvM8/udr\%C5 \%BEitelnost-v-knihovn $\%$ C3 \%A1ch.

27 Quelle: Udržitelnost v knihovnách (knihovna.cz). für das komplette Programm, das schrittweise zusammengestellt wird. Wir laden Fachleute aus verschiedenen Bereichen ein, um unseren eigenen Horizont zu erweitern und gemeinsam Möglichkeiten für die Bibliotheken zu suchen. Auf der Abschlusskonferenz im Dezember werden wir die Ergebnisse vorstellen und Möglichkeiten anschließender Aktivitäten besprechen.

Das Webinar im Januar war eine allgemeine Einleitung ins Thema. Im Februar war das Webinar nur für angemeldete Teilnehmerinnen und Teilnehmer zugänglich, die sich für das ganzjährige Programm angemeldet haben. Bei diesem Treffen ging es um die globalen Zusammenhänge der Problematik nachhaltiger Entwicklung. Am 20. März war der Weltglückstag, daran haben wir bei der Vorbereitung des nächsten Webinars angeknüpft. Wir haben darüber nachgedacht, was Lebensqualität im 21. Jahrhundert bedeutet - speziell im Kontext der Tschechischen Republik - und wie die Bibliotheken darauf einwirken können.

Weitere Webinare werden sich mit den Themen Resilienz oder Büchermarkt beschäftigen, ferner mit praktischen Regeln der Alltagsökologie, der Analyse von Gemeinschaften, nachhaltigen Städten, ökologischer Bildung, Boden und Landwirtschaft. An die UN-Klimakonferenz in Glasgow im November 2021 knüpfen wir mit dem Thema Klimawandel an. Das Programm wird an die Bedürfnisse der Teilnehmer und an aktuelle Ereignisse angepasst.

KN: Wie sind die bisherigen Reaktionen auf die Aktivitäten Ihrer Arbeitsgruppe aus den Reihen der Bibliothekarinnen und Bibliothekare?

NiB: Wir waren ziemlich überrascht, wie positiv die Reaktionen waren. Schon das Seminar, das 2019 stattfand, hatte viele Teilnehmer. Auch die Anzahl an Teilnehmern der oben beschriebenen Online-Umfrage 2019 - es waren fast 350 - hat unsere Erwartungen übertroffen. Die meisten Reaktionen zeigten, dass viele Bibliotheken an dem Thema interessiert sind oder bereits selbst Aktivitäten in Zusammenhang mit dem Thema Nachhaltigkeit realisiert haben. Insgesamt 127 Teilnehmer der Umfrage hinterließen ihre EMail-Adresse, um weiter regelmäßig Informationen zu erhalten. Die Reaktionen auf die bisherigen Weiterbildungsangebote sind auch sehr positiv. Viele Kolleginnen und Kollegen gaben an, dass sie sich gerne an Aktivitäten zum Thema Nachhaltigkeit beteiligen wollen.

KN: Auf welche Hindernisse sind Sie gestoßen? Und wie haben Sie sie gelöst? 
NiB: Ein klassisches Hindernis ist der Mangel an Zeit und Leuten. Die meisten Aktivitäten organisieren wir ehrenamtlich außerhalb unserer regulären Arbeit. Die Arbeitsgruppe besteht aus sieben Personen. Das ist kein Zustand, von dem man Wunder erwarten kann. Trotzdem haben wir relativ viel erreicht - es gibt mittlerweile tolle Materialien, wir haben Partnerschaften geknüpft, die Veranstaltungen laufen gut. Die Begeisterung für die Sache macht natürlich viel aus. Auf jeden Fall wäre es aber gut, für diese Aktivitäten in Zukunft mehr Rückhalt zu haben, was bisher nicht gelang. Wir versuchen, uns Fördermöglichkeiten zu erschließen und werben für die Bildung von Arbeitsgruppen.

Damit hängt eine weitere Herausforderung zusammen - wie kann man die Nachhaltigkeit des Themas Nachhaltigkeit sicherstellen? Das tschechische Bibliothekswesen widmet sich jedes Jahr einem anderen Thema, meistens erlischt danach aber das Interesse, es kommt ein neues Thema, die Materialien dazu verstauben irgendwo. Wir wünschen uns, dass es mit der Nachhaltigkeit anders ist. Auch wenn dieses Thema genuin mit den Bibliotheken verbunden ist, möchten wir, dass einige Aspekte der Nachhaltigkeit vertieft werden und $\mathrm{zu}$ einem untrennbaren Bestandteil der Bibliothekspraxis werden, und dass das Bewusstsein dafür wächst, dass viele Aktivitäten und Veranstaltungen der Bibliotheken die gleiche Ausrichtung haben wie die UN-Nachhaltigkeitsziele. Aus diesem Grund konzentrieren wir uns auf die Weiterbildung von Bibliothekarinnen und Bibliothekaren, auf Informationsverbreitung und Networking.

\section{$K N$ : Was planen Sie für die Zukunft?}

NiB: Wir würden uns wünschen, dass die Bibliotheken ihre einzigartige Position und ihre Möglichkeiten erkennen, die sich ihnen mit dem Thema Nachhaltigkeit eröffnen. Wir würden die Bibliotheken gerne unterstützen, ihnen Materialien und Konsultationen anbieten, sie ermuntern, keine
Angst vor Veränderungen im Betrieb oder vor neuen Themen, vor neuen Herangehensweisen oder Verbindungen zu haben. Wir können aber als kleine Gruppe nicht langfristig ohne Förderung funktionieren. Deshalb hoffen wir, dass für die Aktivitäten im Rahmen der Nachhaltigkeit langfristige Quellen und Unterstützungsmöglichkeiten sichergestellt werden können, damit die Transformation der Bibliotheken in dieser Richtung weiter gehen kann.

Wir wollen das Jahr der Nachhaltigkeit in den Bibliotheken so ausfüllen, dass die Jury des IFLA Green Library Award $2022^{28}$ einen Bericht darüber erhalten kann, und unsere Aktivitäten somit mehr Gewicht erhalten. Es gibt vorläufige Pläne, auch im nächsten Jahr mit dem Weiterbildungsprogramm fortzufahren. Wir glauben, dass die Bibliotheken in der Tschechischen Republik eine gute Grundlage haben und ihre Reichweite in die Gesellschaft groß ist, und deshalb kann auch ihre Rolle bei der Erreichung der Nachhaltigkeitsziele von tragender Bedeutung sein.

\section{Literaturverzeichnis}

Národní knihovna České republiky (Hrsg.) (2020): Koncepce rozvoje knihoven v České republice na léta 2021-2027 s výhledem do roku 2030: knihovny - pilî̌̌e občanské společnosti, vzdělanosti a kultury. Verfügbar unter https://ipk.nkp.cz/docs/koncepce-ro zvoje-knihoven-2021-2027.

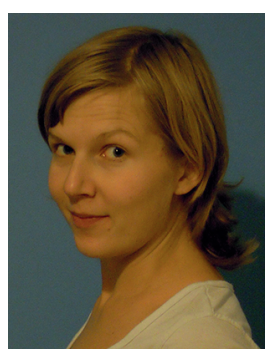

Kateřina Nekolová

Bildungsabteilung des Bibliotheksinstituts Nationalbibliothek der Tschechischen

Republik

Klementinum 190

CZ-110 00 Prag

Tschechien

katerina.nekolova@nkp.cz 